\section{Pincer nail deformity in a patient with amyotrophic lateral sclerosis}

\author{
Youshi Fujita, Takehisa Fujita \\ Fujita Neurological Hospital, Fukui, Japan
}

\section{Abstract}

Although several manifestations of skin involvement in cases of amyotrophic lateral sclerosis (ALS) have been reported, the pincer nail deformity has not been previously reported in a patient with ALS. The pincer nail deformity is characterized by distortion of the shape of the nail with transverse over curvature and may be associated with systemic diseases and certain medications. We herein describe the case of a patient presenting with a pincer nail deformity associated with rapidly advancing ALS. This case suggests a potential link between quickly advancing disease and the pincer nail deformity.

\section{Introduction}

Amyotrophic lateral sclerosis (ALS) is a neurodegenerative disorder affecting motor neurons that is characterized by progressive skeletal muscle atrophy and eventual death, usually due to respiratory failure. Various structural, biological and physiological alterations of the skin have been reported in patients with ALS.1-5 The pincer nail deformity is characterized by distortion of the shape of the nail with transverse overcurvature. ${ }^{6-9}$ This deformity has not been previously reported in patients with ALS. We herein describe the case of a patient who presented with a pincer nail deformity associated with rapidly advancing ALS.

\section{Case Report}

A Buddhist priest began to experience weakness of the right upper and lower limbs at 64 years of age. Six months after onset, a clinical examination revealed weakness in all four limbs [Medical Research Council (MRC) grade 4] with widespread fasciculation and hyperreflexia in the lower extremities. Plantar reflexes were in flexion on both sides, and there were no sensory abnormalities or cognitive disturbances. Magnetic resonance images of the brain and spinal cord were normal; however, electromyography revealed changes indicating acute and chronic denervation in the tongue, facial muscles and all limb muscles. In contrast, motor and sensory nerve conduction studies were normal, as were routine laboratory tests. Based on the revised El Escorial criteria, ALS was confirmed as the clinical diagnosis, and the patient was treated with $100 \mathrm{mg}$ of riluzole daily.

Approximately 13 months after onset, the patient's clinical features rapidly worsened, with progressive weakness in all limbs, predominantly on the right side (MRC grade 2). The rapidly progressive weakness was accompanied by a mild pinching sensation in the fingertips in both hands, although predominantly in the right hand. A physical examination revealed transverse overcurvature of the nails to the extent that the nails were now pinching the fingertips (Figure 1) on both hands, consistent with a pincer nail deformity. Although the mild pinching sensation continued, no treatment was performed due to the patient's lack of pain. He became bedridden at that time. Although an alternating pressure air mattress helped to prevent bed sores, the dysphagia gradually worsened and he was unable to take solid food during the last two months of his life. He subsequently stopped taking riluzole because allowing the medication to dissolve in his mouth induced numbness of the tongue. He thereafter received palliative care, with morphine to combat dyspnea during the last three weeks of his life. The clinical course lasted one year and eight months.

\section{Discussion and Conclusions}

Several skin alterations have been reported in patients with ALS, including elastosis, increased mucopolysaccharides, altered collagen, thickened blood vessel walls and reduced sweat secretion in association with advancing disease. ${ }^{1-5}$ The pincer nail deformity is characterized by distortion of the shape of the nail with transverse overcurvature, resulting in pinching of the soft tissue of the digit. ${ }^{6}$ Pincer nails may be associated with systemic diseases, such as psoriasis, systemic lupus erythematosus, Kawasaki disease, osteoarthritis of the distal phalanx or renal failure, as well as $\beta$-blocker intake. ${ }^{6-9}$ However, there have been no previous reports of ALS patients presenting with pincer nails. Due to the absence of $\beta$ blocker use or systemic disease other than ALS in this patient, we speculate that the nail deformity was related to the advancing ALS.

Compared with that observed in agematched controls, the activity of matrix metalloproteinase (MMP)-9 in the skin is increased in patients with ALS. ${ }^{4}$ An elevated MMP-9 level is associated with more rapid disease progression, ${ }^{4}$ and MMP-9 upregulation may be linked to collagen degradation and shrinking of the
Correspondence: Youshi Fujita, Fujita Neurological Hospital, Hasaki, Maruoka, Fukui 910-0367, Japan.

Tel.: +81.776.671.120 - Fax: +81.776 .670 .282$

E-mail: fujitauc@gmail.com

Key words: amyotrophic lateral sclerosis, skin, pincer nail deformity.

Contributions: YF, clinical work and writing of the manuscript; TF, clinical work and critical review.

Conflict of interests: the authors declare no potential conflict of interests.

Received for publication: 12 November 2014 Accepted for publication: 17 Novmber 2014.

This work is licensed under a Creative Commons Attribution NonCommercial 3.0 License (CC BYNC 3.0).

(C) Copyright Y. Fujita and T. Fujita, 2014

Licensee PAGEPress, Italy

Neurology International 2014; 6:5716

doi:10.4081/ni.2014.5716

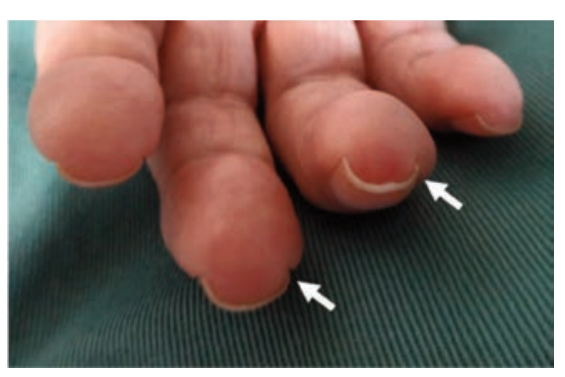

Figure 1. Convex transverse overcurvature of the nails that pinched the fingertips (arrows) 13 months after onset of amyotrophic lateral sclerosis in a patient who experienced rapid deterioration and advancement of the disease

nail bed, which subsequently contributes to nail incurvation. Furthermore, a disturbance in the sympathetic skin response and hypohidrosis on the palm may also occur in those in an advanced stage of ALS, ${ }^{5}$ and the use of $\beta$ blockers, which depress the sympathetic activity, may also induce pincer nail formation. ${ }^{9}$ These observations suggest a potential link between rapidly advancing ALS and the pincer nail deformity, as observed in the current case.

\section{References}

1. Ono S, Toyokura Y, Mannen T, Ishibashi Y. Amyotrophic lateral sclerosis: histologic, histochemical, and ultrastructural abnormalities of skin. Neurology 1986;36:948-56. 
2. Kolde G, Bachus R, Ludolph AC. Skin involvement in amyotrophic lateral sclerosis. Lancet 1996;347:1226-7.

3. Ono S, Imai T, Takahashi $\mathrm{K}$, et al. Decreased type IV collagen of skin and serum in patients with amyotrophic lateral sclerosis. Neurology 1998;51:114-20.

4. Fang L, Huber-Abel F, Teuchert M, et al. Linking neuron and skin: matrix metalloproteinases in amyotrophic lateral sclero- sis (ALS). J Neurol Sci 2009;285:62-6.

5. Beck M, Giess R, Magnus T, et al. Progressive sudomotor dysfunction in amyotrophic lateral sclerosis. J Neurol Neurosurg Psychiatry 2002;73:68-70.

6. Kosaka M, Kusuhara H, Mochizuki Y, et al. Morphologic study of normal, ingrown, and pincer nails. Dermatol Surg 2010;36:31-8.

7. Kirkland CR, Sheth P. Acquired pincer nail deformity associated with end stage renal disease secondary to diabetes. Dermatol Online J 2009;15:17.

8. Vanderhooft SL, Vanderhooft JE. Pincer nail deformity after Kawasaki's disease. J Am Acad Dermatol 1999;41:341-2.

9. Greiner D, Schöfer H, Milbradt R. Reversible transverse overcurvature of the nails (pincer nails) after treatment with a beta-blocker. J Am Acad Dermatol 1998;39: 486-7. 